\title{
Development of a Thrust Stand to Meet LISA Mission Requirements
}

\author{
W. D. Willis III, C. M. Zakrzwski, and S. M. Merkowitz \\ NASA Goddard Space Flight Center \\ Greenbelt, Maryland 20771, USA \\ July 2002
}

\begin{abstract}
A thrust stand has been built to measure the force-noise produced by electrostatic microNewton $(\mu N)$ thrusters. The LISA mission's Disturbance Reduction System (DRS) requires thrusters that are capable of producing continuous thrust levels between 1-100 $\mu \mathrm{N}$ with a resolution of $0.1 \mu \mathrm{N}$. The stationary force-noise produced by these thrusters must not exceed $0.1 \mu \mathrm{N} / \sqrt{ } \mathrm{Hz}$ in the measurement bandwidth $10^{-4}$ to $1 \mathrm{~Hz}$. The LISA Thrust Stand (LTS) is a torsion-balance type thrust stand designed to meet the following requirements: stationary force-noise measurements from $10^{-4}$ to $1 \mathrm{~Hz}$ with $0.1 \mu \mathrm{N} / \sqrt{\mathrm{Hz}}$ sensitivity, absolute thrust measurements from 1-100 $\mu \mathrm{N}$ with better than $0.1 \mu \mathrm{N}$ resolution, and dynamic thruster response from $10^{-4}$ to $10 \mathrm{~Hz}$. The LTS employs a unique vertical configuration, autocollimator for angular position measurements, and electrostatic actuators that are used for dynamic pendulum control and null-mode measurements. Force-noise levels are measured indirectly by characterizing the thrust stand as a spring-mass system. The LTS was initially designed to test the indium FEEP thruster developed by the Austrian Research Center in Seibersdorf (ARCS), but can be modified for testing other thrusters of this type.
\end{abstract}

\section{Introduction}

The Laser Interferometer Space Antenna (LISA) is a collaborative effort between NASA and the European Space Agency (ESA) that is expected to launch in 2011. The LISA mission is to detect gravitational waves produced by galactic binaries and coalescing massive black holes. These waves are expected to be in the range of $10^{-4}$ to $1 \mathrm{~Hz}$. LISA will be composed of three spacecraft that fly in an equilateral triangle and are separated by 5 million kilometers. LISA uses laser interferometers to measure the changes in separation distance between the proof masses of each spacecraft. A gravitational wave is detected when it passes the LISA constellation and causes these distances to expand and contract.

A critical part of the success of LISA depends on being able to maintain precise spacecraft positions for drag-free control and laser pointing. This positioning may be done with the use of Field Emission Electric Propulsion (FEEP) thrusters as part of the LISA Disturbance Reduction System (DRS). The primary goal of these thrusters is to balance the solar radiation pressure exerted on the spacecraft. These thrusters will operate continuously and must not exceed the LISA stationary force-noise requirement of $0.1 \mu \mathrm{N} / \sqrt{\mathrm{Hz}}$ in the measurement bandwidth $\left(10^{-4}\right.$ to $\left.1 \mathrm{~Hz}\right)$.
An indium FEEP thruster developed by the Austrian Research Center in Seibersdorf (ARCS) may be considered for the LISA mission if it can be shown that its noise levels meet the LISA force-noise requirement. ${ }^{1.2}$ The use of a highly sensitive and responsive $\mu \mathrm{N}$ thrust stand can make this measurement.

The LISA Thrust Stand (LTS) is a torsion balance type thrust stand that was developed at NASA Goddard Space Flight Center for the purpose of measuring the thrust and stationary force-noise produced by $\mu \mathrm{N}$ electrostatic thrusters. ${ }^{3}$ Thrust and noise measurements are made indirectly by observing the thrust stands response to thrust input. The first thruster being tested on the LTS is the indium FEEP provided by the ARCS. The LTS was designed specifically to test the indium FEEP thruster, and would have to be modified for testing other thrusters such as the cesium FEEP.

The LTS was designed to make three types of measurements. These measurements characterize the performance of $\mu \mathrm{N}$ electrostatic thrusters and include the following: stationary force-noise measurements below $1 \mathrm{~Hz}$ with $0.1 \mu \mathrm{N} / \sqrt{\mathrm{Hz}}$ sensitivity, absolute thrust measurement from 1-100 $\mu \mathrm{N}$ with better than $0.1 \mu \mathrm{N}$ resolution, and thruster response up to $10 \mathrm{~Hz}$.

This paper discusses the theory and concept for the LTS and describes the current design. Initial calibration measurements are also included with a 
discussion of the stands potential capabilities and expected performance.

\section{Concept and Theory}

A torsion balance is a spring-mass system consisting of a pendulum body (mass) suspended by a fiber (spring). The pendulum body oscillates about the fiber axis, where the twisting of the fiber is providing the restoring force for the oscillations. The behavior of a rotational spring-mass system is defined by the second order differential equation:

$$
\mathrm{I}_{z} \alpha+\mathrm{k}_{\tau} \theta=\tau
$$

where $I_{z}$ is the pendulums mass moment of inertia about the fiber axis, $k_{\tau}$ is the torsion spring constant for the system, and $\tau$ is the external torque provided by the thruster. The $\alpha$ and $\theta$ terms are angular acceleration and position with respect to the fiber axis respectively. Damping is initially considered negligible, but later confirmed by observing the decay of the pendulums free period.

Noise measurements are made indirectly by characterizing the thrust stand as a spring-mass system and correlating angular displacements (dynamic mode) or voltages (null mode) with forces. Stationary forcenoise is defined as random noise that has a normal distribution about the average thrust value. An example of a constant thrust level that contains stationary noise is shown in Figure 1. This thrust produces a torque on the pendulum and causes it to twist about the fiber axis. The response of the pendulum will resemble the oscillations shown in Figure 2 with a period of $T=$ $2 \pi\left[\mathrm{I}_{z} / \mathrm{k}_{\tau}\right]^{1 / 2}$. These large oscillations represent the transient response of the pendulum to the thrust input. The steady-state response of the pendulum to the thrusters force-noise is also present in these oscillations. This noise-response becomes obvious when the natural period of the pendulum is eliminated from the data. The large transient oscillations are damped using the rotation stage that supports the pendulum. Any remaining transient response is subtracted using a numeric notch filter. This filter averages all data points in the signal at $T / 2$ second intervals. If the signal contained no steady state response, the filtered data would represent a constant offset that is consistent with the step input thrust level. Figure 3 shows a theoretical plot of the thrust stand response after the pendulums free period is subtracted from the data. A power spectral density routine is performed on the noiseresponse data. The result is an expression for the steady-state amplitude of the pendulum $(\theta)$ as a function of frequency $(\omega)$.
The steady-state response of a spring-mass system to a sinusoidal forcing is given by:

$$
\theta(\tau, \omega)=\tau /\left|\mathrm{k}_{\tau}-\mathrm{I}_{\mathbf{z}} \omega^{2}\right|
$$

where $\theta$ is the steady state amplitude for a forcing at frequency $\omega$. Torque $\tau$ is equivalent to the moment arm length $L$ crossed with force $F$. This substitution is made in equation (2), and force $F$ is solved for:

$$
F(\theta, \omega)=\theta \mid k_{\tau}-I_{z} \omega^{2} / / L
$$

Equation (3) is used to correlate force amplitudes with steady-state response amplitudes from the power spectral density data. These values will be compared with the LISA requirement as shown in Figure 4.

Average thrust is determined by averaging response data and applying Hook's law: $\mathrm{F}_{\mathrm{ave}}=\mathrm{k}_{\tau} \theta_{\mathrm{ave}} / \mathrm{L}$.

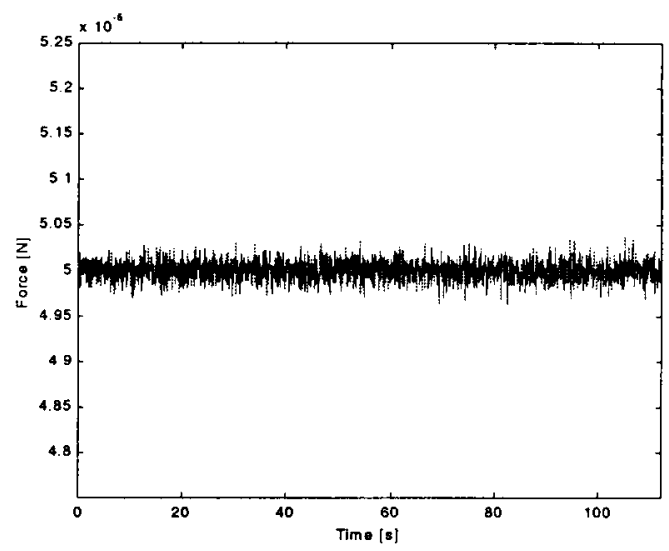

Figure 1: Theoretical Thruster Input Containing Force-Noise

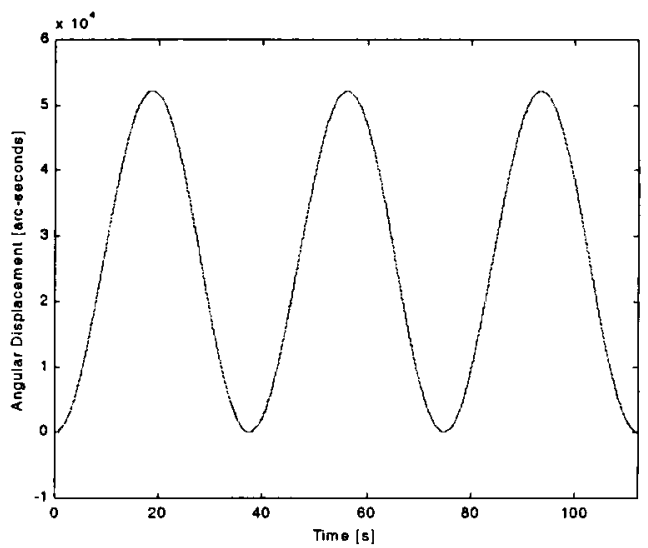

Figure 2: Theoretical Response to Input 


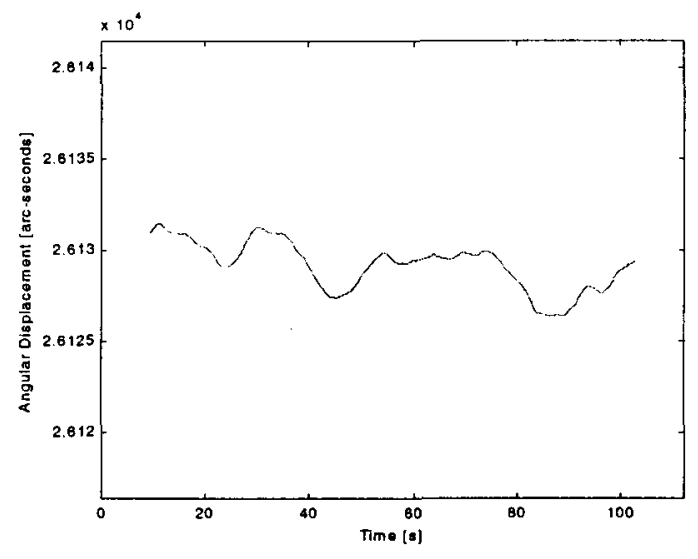

Figure 3: Theoretical Steady State Response

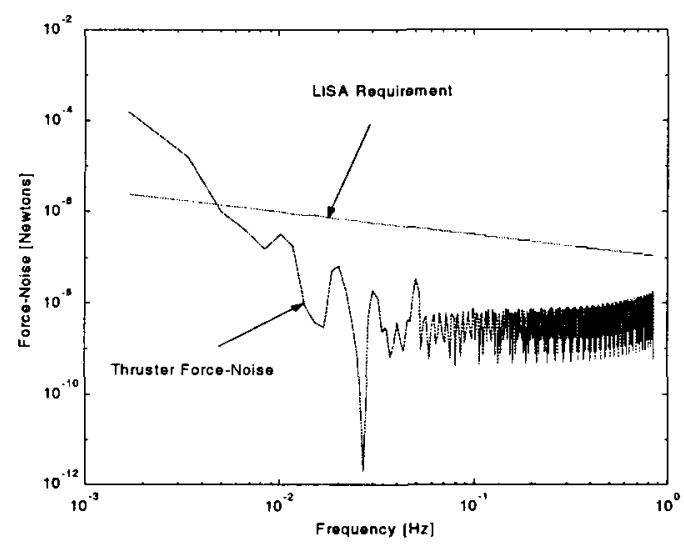

Figure 4: Power Spectral Density of Thruster Force-Noise Plotted Against LISA Requirement

\section{Design Description}

Illustrations of the LTS are shown in Figure 5 and Figure 6. The thrust stand structure shown in Figure 5 is $975 \mathrm{~mm}$ tall and $610 \mathrm{~mm}$ in diameter and is enclosed in a vacuum chamber. Figure 6 shows the location of the FEEP thruster and power module (EMCO) that are attached to the pendulum. The power module is a dcdc, low to high voltage conversion module that supplies the FEEP with $4.5-10 \mathrm{kV}$. The angular position of the pendulum is monitored using an autocollimator (shown in Figure 14) with 0.01 arc-second resolution at $1 \mathrm{~Hz}$. This autocollimator is mounted outside the vacuum chamber and targets a mirror attached to the pendulum body.

The LTS differs from most torsion balance thruststands in that is has a vertical rather than horizontal configuration. The FEEP and EMCO are positioned as close to the fiber axis as possible to minimize the pendulums mass moment of inertia about the vertical axis. The widest part of the pendulum is $120 \mathrm{~mm}$ at its base. Minimizing the pendulums radial profile lowers the pendulums rotational mass and effectively increases its angular acceleration for a given torque: $\alpha=\tau / \mathrm{I}$. The pendulum is therefore more sensitive to fluctuations in thrust, or force-noise than the horizontal type configuration with long moment arms. The height of the pendulum body is $210 \mathrm{~mm}$ from its base to the fiber connection point. This height in the fiber axis direction increases the pendulums stability for pitching and rocking. Therefore, the most sensitive rotation mode for the pendulum is about the vertical, or fiber axis (yaw). The current design is mathematically sensitive to $0.027 \mu \mathrm{N}$ thrust amplitude at $1 \mathrm{~Hz}$ for $\mathrm{I}_{\mathrm{z}}=0.000443$ $\mathrm{kg}-\mathrm{m}^{2}$ (measured).

The pendulum body is supported by a single tungsten fiber. The use of a single fiber has two advantages over using multiple fibers. First, the fiber axis passes through the pendulums center of gravity (CG) making the systems twisting mode relatively insensitive to external vibration. Second, lower torsion spring constants can be achieved with the use of only one fiber. The LTS tungsten fiber has a $100 \mu \mathrm{m}$ diameter and is $533 \mathrm{~mm}$ long. The fibers torsion spring constant is $2.65 \times 10^{-6} \mathrm{Nm} / \mathrm{rad}$ (measured). The fiber diameter was minimized to reduce the stiffness it contributes to the system while still being able to support the weight of the pendulum. The suspended mass is $0.45 \mathrm{~kg}$, resulting in a factor of safety of 3.9 for fiber yield. The fiber is attached at its ends by crimping them inside small copper tubes. These tubes are soldered inside socket-head cap screws that are screwed to the pendulum head and rotation stage.

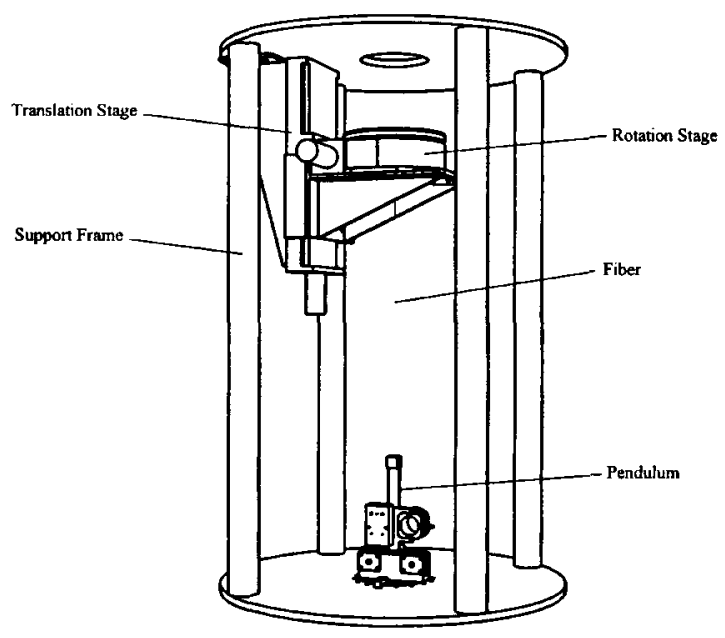

Figure 5: Overview of LTS 


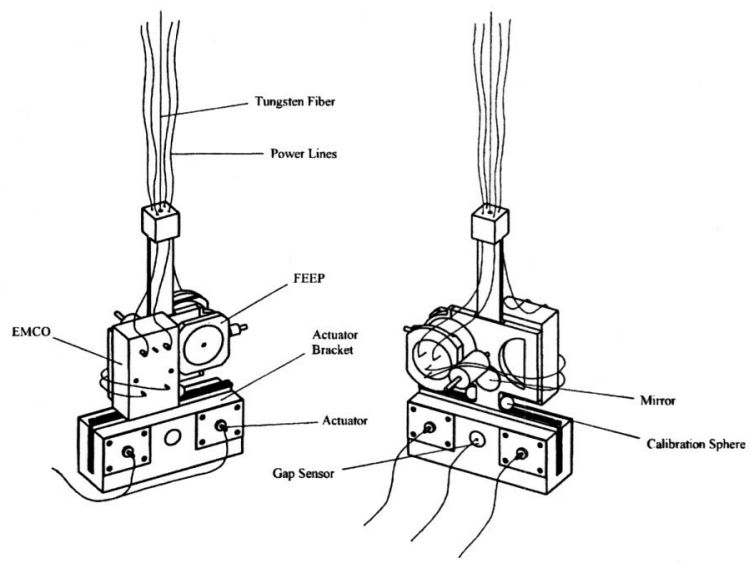

Figure 6: Close Up of Pendulum Showing Front (left) and Back (right)

The upper end of the tungsten fiber is connected to a rotation stage that is connected to a translation stage. The translation stage is used to raise and lower the pendulum. This is useful for 'parking' the pendulum and raising it out of the actuator bracket for balancing and alignment. The rotation stage is used to position the reflected autocollimator beam on the center of the detector (null position).

Low voltage is brought to the pendulum by four 36 gage insulated copper wires. These wires are slack and do not support any of the pendulums weight. Running high voltage to the pendulum with these wires was considered until testing demonstrated electrostatic forces between the copper wires and tungsten fiber exceeded forces in the $\mu \mathrm{N}$ range. This occurred for voltages over $1 \mathrm{kV}$. Therefore, the EMCO is attached to the pendulum and the high voltage is generated on the pendulum body.

The torsion stiffness provided by the four wires was measured to be $9.87 \times 10^{-6} \mathrm{Nm} / \mathrm{rad}$. This is 3.7 times the stiffness of the tungsten fiber. Therefore, the copper power lines dominate the systems stiffness. These wires are attached to the pendulum head $7 \mathrm{~mm}$ from the fiber axis. This distance was minimized to reduce the torque the wires exert by bending when the pendulum twists.

There are two basic modes of pendulum operation. One is a dynamic mode that allows the pendulum to respond freely to the FEEP input. The second mode is a null mode where four electrostatic actuators are used to balance the pendulum in the null position while the thruster is firing. The actuators are calibrated such that electrode voltages are correlated with thrust. These actuators are contained in a bracket that encloses the lower portion of the pendulum as shown in Figure 7 and Figure 8. Each actuator is an electrode that has a flat,
$20 \mathrm{~mm}$ diameter circular face that is positioned $2 \mathrm{~mm}$ from and parallel to the pendulum surface.

Applying a potential of $0-500$ volts to the electrodes will attract the pendulum body (ground) by an electrostatic force:

$$
F=\varepsilon_{o} V^{2} A / 2 d^{2}
$$

where $F$ is force, $\varepsilon_{o}$ is permittivity of free space, $V$ is voltage, $\mathrm{A}$ is the surface area of the electrode, and $\mathrm{d}$ is the gap between the electrode and ground. A coupled moment acts on the pendulum by charging two electrodes at opposite corners of the bracket (electrodes 1 and 4). Swing in the X-direction can be taken out of the pendulum by charging two electrodes on one side of the pendulum (electrodes 1 and 2). Each electrode can also be operated independently.

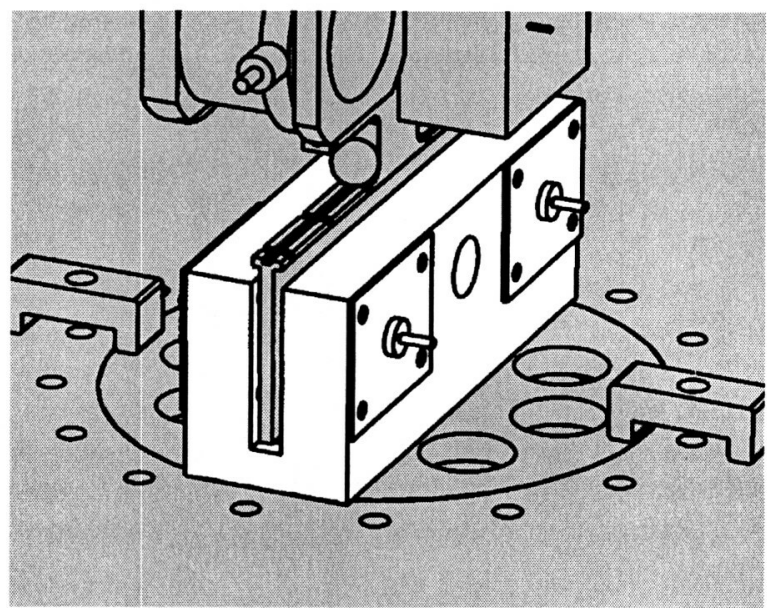

Figure 7: Illustration Showing Lower Portion of LTS Hanging Freely in the Actuator Bracket

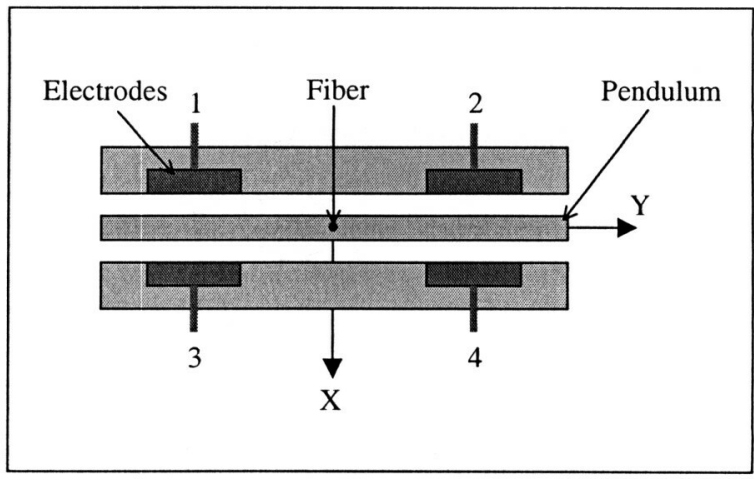

Figure 8: Electrostatic Actuators and Bracket (Top View)

The null mode is achieved by feeding back the signals from the autocollimator and capacitive gap sensor. The capacitive gap sensor is also attached to 
the actuator bracket and monitors the position of the pendulum in the X-direction (normal to electrode face). The null mode has the advantage over the dynamic mode of increasing the pendulums measurement bandwidth. This is achieved by using the actuators to keep the LTS in the autocollimator range. The dynamic mode has a more limited range because low frequency (less than the LTS natural frequency) force-noise may produce angular displacements that are out of this range. The null mode has another advantage over the dynamic mode because the actuators can provide infinite stiffness to the system. Therefore, the systems spring constant does not become a factor and errors associated with the LTS natural frequency are avoided.

A Finite Element Method (FEM) program was used to perform a coupled-field analysis of the actuator system. The electric field created by the electrodes was modeled to minimize the fringe fields and to predict the applied torque on the pendulum. Table 1 summarizes some of the results from the analysis. All these values are for the null position where there is no pendulum twist or swing. The torque column represents the torque that one electrode applies to the pendulum for a given voltage. The Balance Thrust column represents the thrust level that will produce the same torque for a given electrode voltage. For example, a constant thrust value of $17.6 \mu \mathrm{N}$ would require 200 volts to balance the LTS in the null position.

Figure 9 shows the relationship between torque and Y-forces caused by fringe fields. The plotted fringe force values are in the direction that pulls the pendulum out of the actuator bracket (Y-direction, parallel to electrode face, see Figure 8). The plot values on the horizontal axis represent the position of the electrode. The vertical axis gives the $\mathrm{Y}$-force and torque values for one electrode at 300 Volts. Moving the electrode along the pendulum, away from the fiber axis, increases the torque applied by the electrode. These values increase until the electrode starts to overhang the pendulum $(>50 \mathrm{~mm})$. This is also the position where the Y-forces increase dramatically. The results from this analysis were used to choose a conservative electrode position of $40 \mathrm{~mm}$.

Two counterweights are used to balance the pendulum for pitch and roll. They are cantilevered horizontally from the pendulum body in the $\mathrm{X}$ and $\mathrm{Y}$ direction. See Figure 10. Their axes lie in the same horizontal plane as the pendulums center of gravity. Each counterweight consists of a nut and threaded rod. Pendulum balance is maintained by advancing the nuts in the appropriate direction. The counterweight in the $\mathrm{X}$-direction balances pendulum tilt in the Y-Z plane, and the counterweight in the $\mathrm{Y}$-direction balances tilt in the $\mathrm{X}-\mathrm{Z}$ plane.
Table 1: ANSYS Torque Values

\begin{tabular}{|c|c|c|}
\hline Voltage & Torque $[\boldsymbol{\mu N m}]$ & Balance Thrust $[\boldsymbol{\mu N}]$ \\
\hline 50 & 0.035 & 1.1 \\
\hline 100 & 0.139 & 4.4 \\
\hline 200 & 0.557 & 17.6 \\
\hline 300 & 1.252 & 39.5 \\
\hline 500 & 3.480 & 109.7 \\
\hline
\end{tabular}

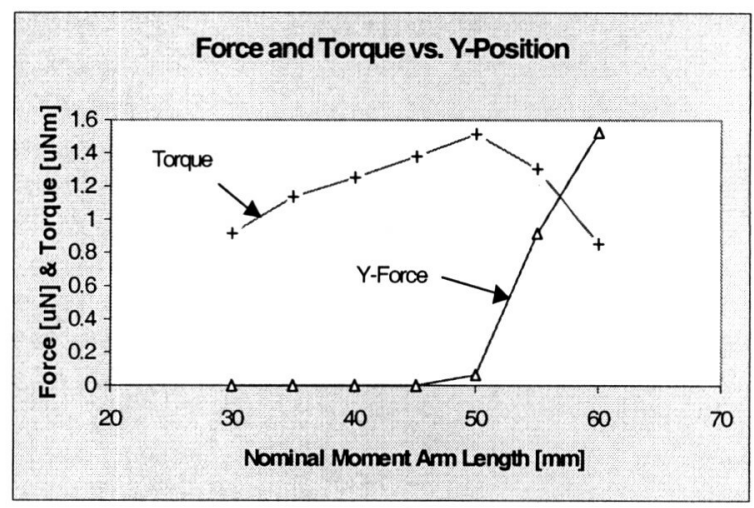

Figure 9: Plot Showing Relationship Between Fringe Forces and Torque

The counterweights contribution toward the pendulums moment of inertia $I_{z}$ was minimized. Mass moment of inertia is proportional to mass and distance squared: $I=r^{2} \mathrm{dm}$. Therefore, the counterweight mass was increased to decrease its radial distance from the fiber. This decreases the counterweights moment of inertia $I_{z}$ without loosing its effectiveness for balancing the pendulum.

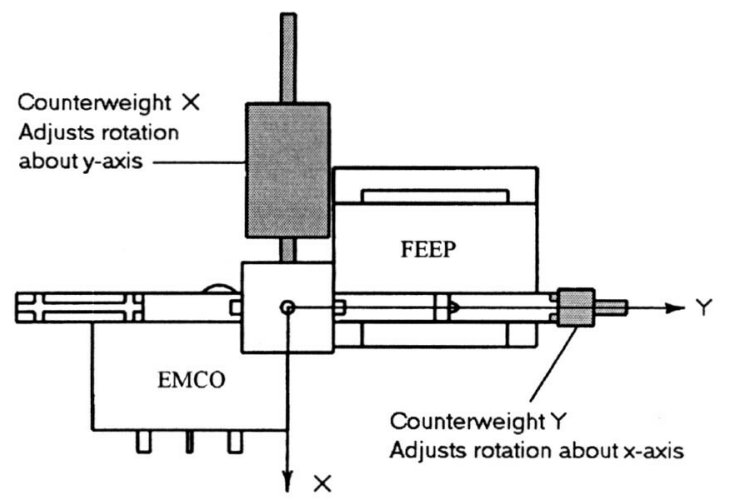

Figure 10: Top View of LTS Showing Location of Counterweights 
A modal analysis was performed on the pendulumfiber system to obtain a better understanding of its behavior. All mode shapes and frequencies were extracted that do not involve structural deflections of the pendulum body. A mode that involves a combination of rolling and pitching may cause the autocollimators light trace on the detector to have a horizontal component. This horizontal component will be read as a false angular displacement. Each mode shape in the measurement bandwidth of $10 \mathrm{~Hz}$ was investigated for angular measurement error potential.

Table 2 shows the results of the modal analysis. Mode 1 is the twisting mode of the pendulum. Modes 2 and 3 are the swinging modes. Modes 4 and 5 involve pendulum rocking about horizontal axes that define the second and third principal moments of inertia. See Figure 11. Mode 6 is similar to modes 4 and 5. Mode 7 is pendulum heaving or bounce in the fiber axis direction. Mode shapes are shown in Figure 12.

Table 2: LTS Modal Analysis Results

\begin{tabular}{|c|c|l|}
\hline Mode & Frequency $[\mathrm{Hz}]$ & \multicolumn{1}{|c|}{ Shape } \\
\hline 1 & 0.0692 & Twist about fiber axis \\
\hline 2 & 0.632 & Swing (front to back) \\
\hline 3 & 0.632 & Swing (side to side) \\
\hline 4 & 3.94 & Rocking about $\mathrm{I}_{3}$ \\
\hline 5 & 5.20 & Rocking about $\mathrm{I}_{2}$ \\
\hline 6 & 7.97 & Rocking between $\mathrm{I}_{2}$ and $\mathrm{I}_{3}$ \\
\hline 7 & 23.2 & Bounce in $\mathrm{z}$-direction \\
\hline
\end{tabular}
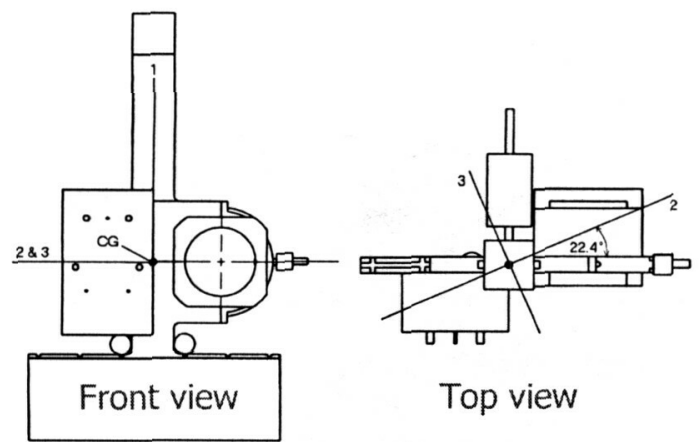

Top view

Figure 11: Location of Principal Moments of Inertia: $I_{\mathbf{1}}$ (Front View, Vertical) $I_{2}$ and $I_{3}$ (Top View)

The modes of concern are modes 4,5 and 6 . These modes are above $1 \mathrm{~Hz}$ and are not expected to introduce significant angular measurement errors.
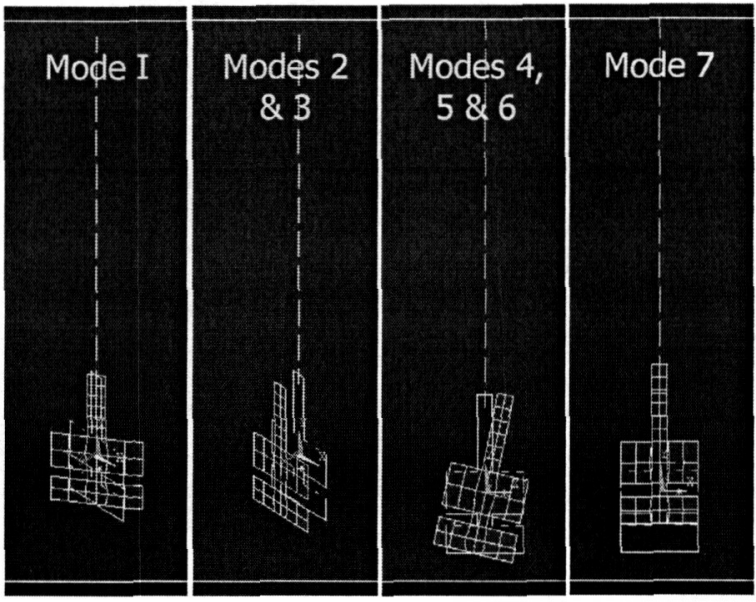

Figure 12: LTS Mode Shapes

\section{Calibration}

The LTS is calibrated by measuring the pendulums natural frequency, mass moment of inertia $I_{z}$, and moment arm length. The mass moment of inertia $I_{z}$ is determined by measuring the difference in pendulum periods caused by moving the two calibration spheres shown in Figure 6. These steel spheres are $10 \mathrm{~mm}$ in diameter and have a mass of $8.19 \mathrm{~g}$. The pendulums free period (T1) is timed with the spheres in the inside position close to the fiber axis. A second period (T2) is timed after moving the spheres $40 \mathrm{~mm}$ outward in a radial direction from the fiber axis. The pendulums moment of inertia is then determined by using the following equation:

$$
\mathrm{I}_{\mathrm{z}}=\mathrm{mr}^{2} \mathrm{~T} 1^{2} /\left(\mathrm{T} 2^{2}-\mathrm{T} 1^{2}\right)
$$

where $\mathrm{m}$ is the mass of the two spheres, and $\mathrm{r}$ is the distance the spheres are moved $(40 \mathrm{~mm})$. Spring constants are calculated using the relationship between a systems moment of inertia and period: $\mathrm{k}_{\tau}=4 \pi^{2} \mathrm{I} / \mathrm{T}^{2}$.

The electrostatic actuators are calibrated using the measured spring constant of the tungsten fiber. The actuators produce a coupled moment on the pendulum causing it to twist. The rotation stage is used to return the pendulum to the null position. The torque provided by the actuators is equal to the torque provided by the tungsten fiber, where the tungsten fiber is applying $k_{\tau} \theta$ $\mathrm{Nm}$. A calibration curve is constructed for voltages up to $500 \mathrm{~V}$.

The moment arm length is defined as the shortest distance between the fiber axis and the thrust vector. This length must be known for determining the force that produces the measured torque: $\mathrm{F}=\tau / \mathrm{L}$, where $\tau$ is torque, $\mathrm{F}$ is force, and $\mathrm{L}$ is the moment arm length. This distance is measured with $0.02 \mathrm{~mm}$ accuracy using 
a transit that has a built in micrometer and a cross-slide. The transit sights down the fiber axis (rotation axis) to the pendulums center of gravity and then measures the horizontal distance to the center of the thrusters nozzle. This measurement technique determines the moment arm length with $99.94 \%$ accuracy, assuming the thrust vector is collinear with the nozzle axis. This accuracy is directly proportional to the accuracy of the force calculation using the $\tau=L F$ equation. The errors associated with the cross-slide path not being parallel to the actual moment arm are small. This error is related to the cosine of the angle between the moment arm and cross-slide path. Therefore, misalignments as large as $5^{\circ}$ will only add $0.4 \%$ error to the moment arm measurement.

\section{$\underline{\text { Facilities }}$}

The vacuum chamber that contains the thrust stand is $1.1 \mathrm{~m}$ high, and $0.75 \mathrm{~m}$ in diameter. It is capable of reaching vacuum levels of $10^{-7}$ torr. The chamber base is bolted to a large support structure that contains the pumps and valves. See Figure 13.

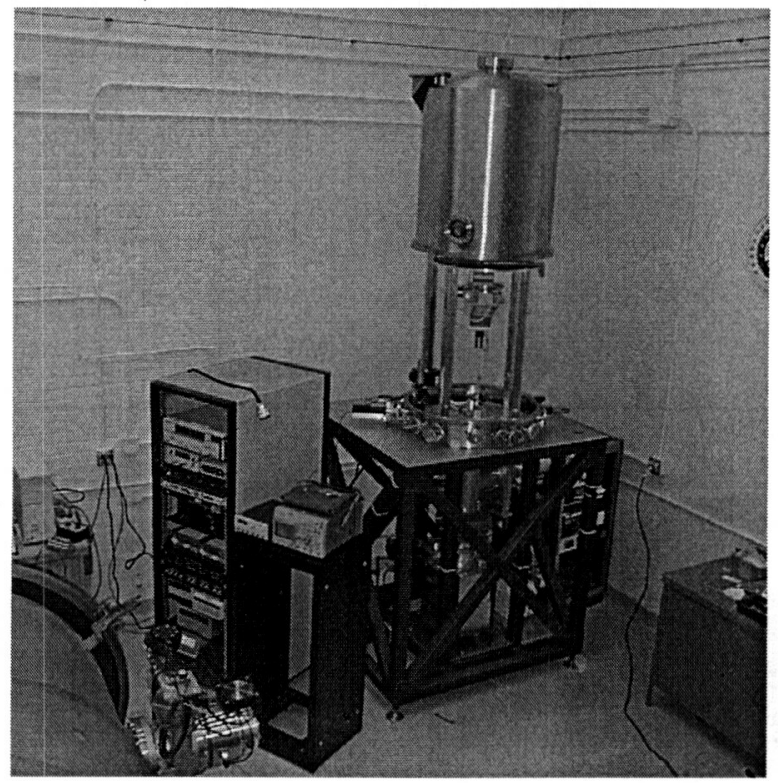

Figure 13: Laboratory Setup Showing the Open Vacuum Chamber Containing the LTS

The support structure was designed to be rigid and not amplify low frequency vibration from the ground to the pendulum. It is constructed of 3 in. square steel tube with $1 / 4$ inch wall thickness and weighs $1650 \mathrm{lb}$. It holds the weight of the bell-jar vacuum chamber and all the vacuum pumps, which weight approximately 1000 lb. A modal analysis was performed on the support structure to ensure its fundamental frequencies were above $100 \mathrm{~Hz}$.

The vacuum pumps include a rough pump, turbo pump and an ion pump. The ion pump does not produce any mechanical noise or vibration and is used to maintain vacuum during FEEP and thrust stand operation.

Figure 14 shows the LTS pendulum inside the open vacuum chamber. The autocollimator is the black box shown in the foreground. It is attached to a shelf that is bolted to a port on the base of the chamber. The glass in this port will reflect a small fraction of the autocollimators light beam. Therefore, the glass is tilted $3^{\circ}$ to ensure the reflected light does not interfere with the autocollimator reading.

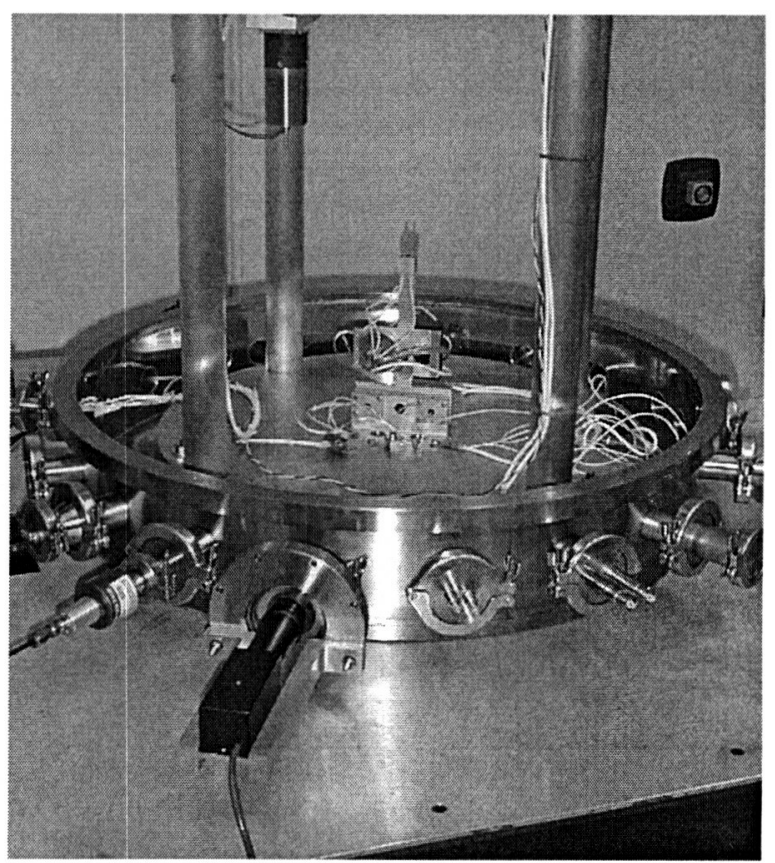

Figure 14: View of LTS in Open Vacuum Chamber

A carbon nano-tube collector plate (not shown) is positioned inside the vacuum chamber in front of the thruster. This plate is semi-cylindrical shaped and is used to collect the ion beam emitted from the FEEP thruster. This beam is collected for two reasons. The first is to minimize contamination of the thruster from back sputter. The second is measure the beam current for calculating the theoretical thrust produced by the thruster. These values are used as a check for the actual thrust measurements made by the pendulum. 


\section{Predicted Performance}

The LTS was mathematically modeled as a single degree of freedom, spring-mass system without damping. The system was characterized by measuring the following parameters: moment of inertia $\mathrm{I}_{z}$, system torsion spring constant $k_{\tau}$ and the moment arm length L. These values are summarized in Table 3 .

Table 3: LTS Parameters

\begin{tabular}{|l|l|c|}
\hline Parameter & Value & Error \\
\hline Mass moment of inertia $\mathrm{I}_{\mathbf{z}}$ & $0.000443 \mathrm{kgm}^{2}$ & $1.4 \%$ \\
\hline Torsion spring constant $\mathrm{k}_{\tau}$ & $12.52 \times 10^{-6} \mathrm{Nm} / \mathrm{rad}$ & $1.4 \%$ \\
\hline Moment arm length $\mathrm{L}$ & $0.03172 \mathrm{~m}$ & $0.06 \%$ \\
\hline
\end{tabular}

Equation (1) was solved for a $0.1 \mu \mathrm{N}$ sinusoidal forcing. Figure 15 shows the pendulums steady-state response that corresponds with the dynamic mode. The LTS natural frequency and period are $0.027 \mathrm{~Hz}$ and 37.37 seconds respectively. This natural frequency lies in the LISA measurement bandwidth and therefore divides the useful measurement range in the dynamic mode. The response of the LTS for frequencies less than $0.003 \mathrm{~Hz}$ is about 52 arc-seconds for the $0.1 \mu \mathrm{N}$ forcing. The response of the pendulum is more dependent on amplitude than forcing frequency at these lower frequencies. The LTS response for frequencies greater than the natural frequency is attenuated as the forcing frequency increases. The response at $1 \mathrm{~Hz}$ for the $0.1 \mu \mathrm{N}$ forcing is 0.0374 arc-seconds. Measurements will not be possible in the dynamic mode at the LTS natural frequency. This is because 0.1 $\mu \mathrm{N}$ force-noise levels at these frequencies will force the LTS out of the autocollimators measurement range ( \pm 600 arc-seconds). The largest force-noise level that can be measured below the LTS natural frequency is 1 $\mu \mathrm{N}$. Force amplitudes larger than this will also force the LTS out of the autocollimators measurement range. This $1 \mu \mathrm{N}$ measurement limit for lower frequencies is lower than the LISA force-noise requirement of 0.1 $\mu \mathrm{N} / \mathrm{JHz}$. Therefore the dynamic mode has a more limited range than the null mode. The range of the null mode is only limited by the resolution of the autocollimator.

The noise floor (background noise) is also a concern and will affect the measurement capabilities. Early attempts to measure the fixed position of the pendulum indicated linear drifting of about 0.01 arcseconds per second. This occurs without powering any components on the pendulum. This drifting was observed after a pendulum settling time of about 30 minutes. More severe drifting is expected once the FEEP is powered. Thermal issues have not been fully addressed and are expected to cause the majority of the measurement difficulties.

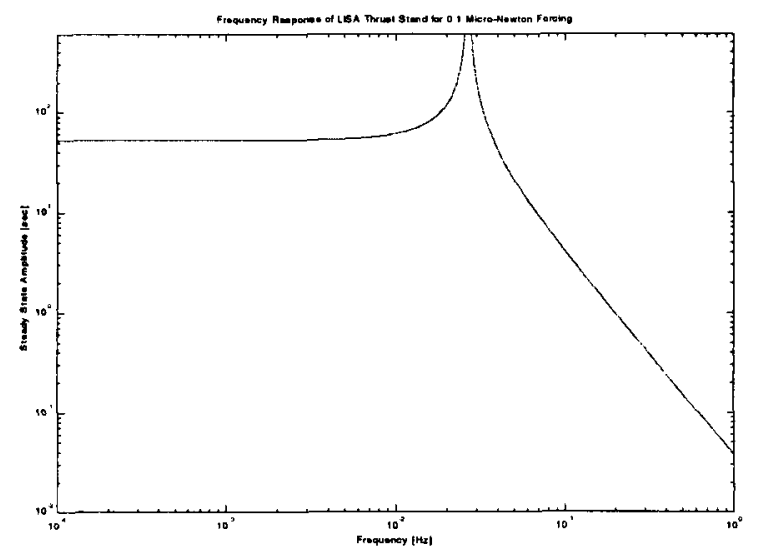

Figure 15: LTS Frequency Response

Another issue involves the electric fields around the LTS generated by high voltage. The pendulums wiring is shielded to reduce the electrostatic forces caused between the high voltage surfaces and ground. The face of the FEEP thruster (emitter plate) is a high voltage surface that cannot be shielded. It is not yet known what the magnitude of the forces will be between this emitter plate and the surrounding ground.

The four power lines are another concern because of their large contribution toward the systems stiffness. This stiffness depends of the position of the wires that change easily. Therefore, their behavior is difficult to define, and these uncertainties will affect thrust measurements.

Uncertainties in the force-noise calculation are determined by propagation of errors. Force is measured indirectly by assuming the LTS behaves as a springmass system defined by equation (1). Forces are correlated with displacements using equation (3). Each term in equation (3) contains calibration errors that are propagated to the force calculation. The accuracy of the force calculation depends on the measurement accuracy of the autocollimator, moment arm length, sphere masses and position, and timing. The error for $0.1 \mu \mathrm{N}$ force amplitude is plotted as a function of frequency in Figure 16. The calibration errors used in the error propagation are shown in Table 4.

Force-noise calculations in the dynamic mode can be made with less than 5\% error for the following frequency ranges: $0-0.02$ and $0.04-0.4 \mathrm{~Hz}$. These values assume the noise levels do not exceed the 
autocollimators range and there is no external noise that would affect the angular measurements.

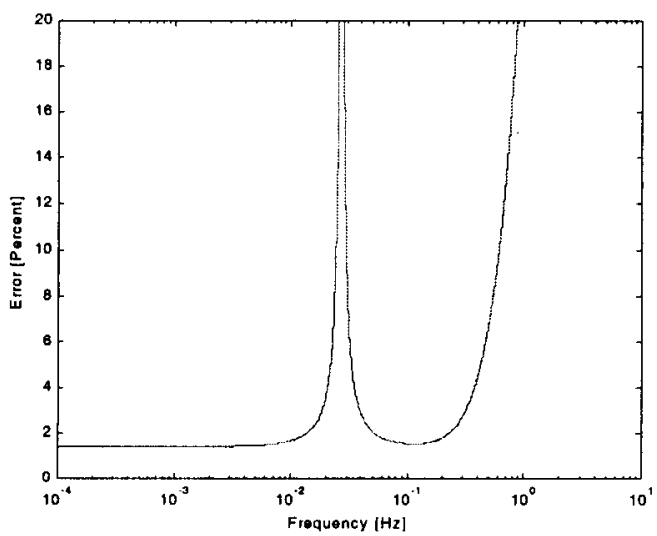

Figure 16: Error in Thrust Calculation vs. Frequency

Table 4: Errors

\begin{tabular}{|l|l|}
\hline Parameter & Error \\
\hline Autocollimator & \pm 0.01 arc-seconds \\
\hline Moment arm length & $\pm 0.00005 \mathrm{~m}$ \\
\hline Sphere mass & $\pm 0.00001 \mathrm{~kg}$ \\
\hline Sphere position & $\pm 0.0001 \mathrm{~m}$ \\
\hline Timing & \pm 0.025 seconds \\
\hline
\end{tabular}

Measurement errors increase rapidly above $1 \mathrm{~Hz}$ because the response amplitudes quickly approach the autocollimator resolution of 0.01 arc-seconds. There are also large errors associated with making measurements near the LTS natural frequency in the dynamic mode. This is due to calibration error where small uncertainties in the natural frequency of the pendulum propagate into large errors when calculating force-noise at these frequencies.

Null mode errors are expected to be consistent with the dynamic mode errors at high frequencies. This is because the null mode uses the autocollimator in the feedback loop. Again, the resolution of the autocollimator is approached for higher frequencies and the autocollimator will not be able to provide an adequate feedback signal for the actuators. The accuracy of lower frequency measurements depends on the accuracy of the systems spring constant measurement. This error is currently $1.4 \%$, and therefore, the actuators should be accurate to $98.6 \%$ for frequencies lower than $0.1 \mathrm{~Hz}$.

\section{$\underline{\text { Conclusions }}$}

The LTS has a vertical configuration that makes it more suited for measuring force-noise than typical horizontal type torsion balances. The combination of the autocollimator and LTS moment of inertia and spring constant makes it theoretically sensitive to $0.027 \mu \mathrm{N}$ at $1 \mathrm{~Hz}$. The measurement range of the LTS is increased by the use of electrostatic actuators. These actuators are used in a null mode and require a smaller portion of the limited range of the autocollimator. The LTS has not yet been tested, but the initial design analysis looks promising. The LTS is expected to characterize the indium FEEP thruster for the LISA force-noise requirements. Work is in progress to address issues that are expected to cause measurement difficulties. Some of this work includes thermal and electrostatic shielding. Overall, this concept design may be well suited to measure $\mu \mathrm{N}$ level thrust and force-noise produced by a variety of thrusters.

\section{References}

1. J. Mitterauer, "Indium: An alternative Propellant for FEEP Thrusters", Paper no. AIAA 2001-3792, $37^{\text {th }}$ Joint Propulsion Conference, Salt Lake City, Utah, USA

2. M. Tajmar, W. Steiger, A. Genovese, "Indium FEEP Thruster Beam Diagnostics, Analysis and Simulation", Paper no. AIAA 2001-3790, $37^{\text {th }}$ Joint Propulsion Conference, Salt Lake City, Utah, USA.

3. S. M. Merkowitz, P. G. Maghami, A. Sharma, W. D. Willis, C. M. Zakrzwski. "A $\mu$ Newton thrust-stand for LISA", Classical and Quantum Gravity, 19 (2002) 1745-1750 